\title{
THE CAUSES OF ADVERSE TREATMENT RESULTS AND THE WAYS OF THEIR ELIMINATION IN BLEEDING FROM CHRONIC GASTRODUODENAL ULCERS
}

DOI: 10.36740/WLek202009211

\author{
Gennady E. Samoilenko, Stanislav 0. Zharikov, Ruslan P. Klimanskyi \\ DONETSK NATIONAL MEDICAL UNIVERSITY, LYMAN, UKRAINE
}

\begin{abstract}
The aim: Is to determine the main causes of adverse outcomes of the patients' treatment with acute ulcerative gastroduodenal bleeding and to develop preventive measures to improve the quality of the patients' treatment with this pathology.

Materials and methods: A retrospective analysis of the treatment results of 1323 patients with bleeding of ulcerative etiology has been carried out. There are 375 patients with gastric ulcer (28.3\%) and 948 patients (71.7\%) with duodenal ulcer among them. The patients' age ranged from 15 to 93 years old. Concomitant pathology was observed in $623(47.1 \%)$ patients, the most common of which were coronary heart disease, chronic non-specific lung diseases and cerebrovascular diseases. Mild severity of blood loss was detected in 404 (30.5\%) patients, moderate severity $693(52.5 \%)$, severe 145 (10.9\%) and extremely severe 81 (6.1\%). Overall mortality was 5.9\%, postoperative mortality $6.3 \%$, the mortality in conservative treatment only $6.1 \%$.

Results: The main cause of the patients' mortality with acute ulcerative gastroduodenal bleeding was decompensated hemorrhagic shock which developed at the prehospital stage in $45.3 \%$ and as a result of bleeding recurrence during treatment $44.2 \%$ of the patients. Inadequate drug therapy increases the risk of bleeding recurrence from 15.8 to $32.7 \%$. The use of proton pump blockers and the combination of bolus and prolonged use of proton pump blockers allow to reduce the risk of bleeding recurrence by half (up to $6.8 \%$ ). Conclusions: Endoscopic hemostasis in combination with the injection method with diathermocoagulation or thermal coagulation can be considered as full-fledged, and when performing endoscopic monitoring the preference should be given to coagulation methods of diathermo- and hydrodietermocoagulation.
\end{abstract}

KEY WORDS: peptic ulcer, bleeding recurrence, diagnosis, treatment, endoscopic hemostasis

Wiad Lek. 2020;73(9 p. II):1957-1961

\section{INTRODUCTION}

The problem of acute ulcerative gastroduodenal bleeding (AUGDB) has been an urgent public health problem for more than 150 years. According to the decision of Maastricht group despite the fact that specific eradication therapy of Helicobacter pylori has been introduced into daily medical practice widely, the number of complications of peptic ulcer in the form of bleeding has a steady upward trend. So, according to the Ministry of Health of Ukraine the largest increase in the cases of ulcerative bleeding in our country has occurred over the past 5 years $[1,2,3]$.

Indeed, there is a decrease in the total number of surgical interventions for peptic ulcer disease but it primarily applies to planned surgical interventions for the treatment of peptic ulcer. Despite significant progress in the development of endoscopic and pharmacological technologies the proportion of the operations to stop ulcer bleeding remains constant even in developed countries of the world. According to some foreign and native authors there is a change in nosological forms in bleeding from the upper gastrointestinal tract - displacing of other diseases due to the growth of ulcerative bleeding from $(50.5 \pm 7.2)$ to $(63.7 \pm 8.4) \%$ of the cases $[4,5,6]$.

Over the past 10 years thanks to the rapid development of endoscopic technologies in our country and CIS countries significant differences have been revealed in the clinical and endoscopic characteristics of ulcer bleeding compared with the countries of Europe and America, the Near and Far East. Thus, according to American scientists' data there has been an increase in the number of the cases with complicated course of peptic ulcer by $20 \%$ over the past decade. 20 million people suffer from peptic ulcer in the United States and about 100000 patients are operated on annually and 6000 people die from various complications of this severe systemic disease $[7,8,9]$.

\section{THE AIM}

The aim of the study is to determine the main causes of adverse outcomes in the patients' treatment with AUGDB and to develop preventive measures in order to improve the quality of the patients' treatment with this pathology.

\section{MATERIALS AND METHODS}

To achieve the goal of the study we carried out the retrospective analysis of the results of the treatment of 1323 patients with bleeding of ulcer etiology who were admitted to the surgery and endoscopy clinic of Donetsk National 
Table I. The main indicators of the patients' treatment with acute ulcerative gastroduodenal bleeding for the period from 2015 to 2018

\begin{tabular}{|c|c|c|c|}
\hline \multirow{2}{*}{ Main characteristics } & \multirow{2}{*}{ The patients' number (\%) } & \multicolumn{2}{|c|}{ Died } \\
\hline & & Abs. & $\%$ \\
\hline A total of the patients: & $1323(100 \%)$ & 79 & $5,9 \%$ \\
\hline stomach ulcer & $375(28.3 \%)$ & 32 & $8,5 \%$ \\
\hline duodenal ulcer & $948(71.7 \%)$ & 47 & $4,9 \%$ \\
\hline Operated: & $268(20.3 \%)$ & 17 & $6,3 \%$ \\
\hline at the height of bleeding & $16(5.9 \%)$ & 3 & $18,8 \%$ \\
\hline due to bleeding recurrence & $52(19.5 \%)$ & 8 & $15,4 \%$ \\
\hline due to unstable hemostasis & $79(29.5 \%)$ & 5 & $6,3 \%$ \\
\hline Planned before & $121(45.1 \%)$ & 1 & $0,8 \%$ \\
\hline Not operated & 1055 (79.7\%) & 64 & $6,1 \%$ \\
\hline
\end{tabular}

Medical University at the central city clinical hospital in Druzhkovka for the period from 2015 to 2018. The patients' age ranged from 15 to 93 years old. At the age of 20 years there were 39 (2.9\%) patients, from 20 to 45 years old 428 (32.4\%), from 46 to 60 years old 380 (28.7\%), from 61 to 74 years old $372(28.1 \%)$ and over 75 years old 104 (7.9\%).

A significant relationship was found between chronic ulcerative bleeding and the presence of concomitant pathology while analyzing concomitant pathology. A combination of two or more diseases was observed in 623 (47.1\%) patients with concomitant pathology, the most common of which were coronary heart disease, chronic non-specific lung diseases, cerebrovascular diseases and diabetes mellitus. In addition, there were $34(5.5 \%)$ patients with decompensated liver cirrhosis with the manifestations of liver failure and 15 (2.4\%) patients with chronic renal failure in this group.

Overall mortality was $5.9 \%$, postoperative mortality $6.3 \%$, mortality with conservative treatment alone $6.1 \%$.

The duration of the prehospital period ranged from 1 hour to 21 days. In the first 24 hours from the onset of the first signs of bleeding 721 (54.5\%) patients were admitted, in the period from 25 to 72 hours 210 (15.9\%), more than 72 hours 389 (29.4\%). Mild severity of blood loss was detected in $404(30.5 \%)$ patients, moderate severity $693(52.5 \%)$, severe $145(10.9 \%)$ and extremely severe 81 (6.1\%). The ulcer was localized in $375(28.3 \%)$ patients in the stomach, and in the duodenum in 948 (71.7\%).

The main indicators of the patients' treatment with AUGDB are presented in table I.

\section{RESULTS}

Analyzing the reasons for unsatisfactory results of the patients' treatment several factors should be noted, the influence of which undoubtedly led to a lethal outcome. Unfortunately, not all the components of the course of the disease were able to be influenced actively. To facilitate the systematization of adverse factors it was decided to divide them into two main categories: objective and subjective.

The duration of the prehospital period, the intensity of bleeding, the degree of the compensation of concomitant pathology, topographic features of the source of bleeding and also such a factor as the patient's refusal to undergo treatment (both drug and endoscopic) were referred to the first category of the factors that we were not able to influence.

The category of subjective factors includes the diagnostic value of the primary endoscopic examination, medical manipulations both at the first stage and in the process of medical monitoring, the compliance with tactical settings and the usefulness of drug therapy.

Contrary to the expectations the continuation of the prehospital stage did not affect the unfavorable prognosis of the course of the disease significantly. All things being equal, the mortality rate among the patients hospitalized in the first 12 hours from the onset of the first signs of bleeding was the highest (8.5\%) and as the time increased it had a clear tendency to decrease to $4.8 \%$ after 48 hours. This factor, perhaps, cannot be decisive in terms of predicting an adverse outcome of the disease. The degree of blood loss had a significant effect. There was a steady increase in mortality from $0.7 \%$ with mild blood loss to $39 \%$ with extremely severe blood loss.

Acute gastroduodenal ulcers associated with the drug usage were diagnosed in 251 patients (18.9\%) and recently accounted for a third of the causes of gastroduodenal bleeding in elderly patients. According to our materials it is the second most frequent cause of the complications of acute ulcers in inpatients. For the most part, these ulcers appear after taking cyclooxygenase inhibitors. In this group there were 159 patients $(63.3 \%)$ aged over 65 years old with cardiovascular disease and they took more than 3 drugs a day, usually disaggregants (acetylsalicylic acid), anticoagulants (warfarin), non-steroidal anti-inflammatory drugs ( diclofenac). The combination of dyspepsia symptoms with the constant use of disaggregants increases the risk of developing acute ulcers in 2 times. In addition, we also included the risk factors for the development of such gastropathies as old age, the presence of ulcerative history, decompensated forms of concomitant diseases (cardiovascular, cerebrovascular, diabetes mellitus), decreased physical activity, 
simultaneous taking of anticoagulants, glucocorticosteroids and non-steroidal anti-inflammatory drugs.

The risk of the development of erosive and ulcerative lesions of the gastrointestinal tract increases in 2 times with the combination of 2 or more of these factors. In $75-90 \%$ of cases they are complicated by gastrointestinal bleeding.

Thus, having analyzed the patients' data of the study group we attributed old age, decreased physical activity, II-III degree of organ dysfunction according to the severity of APACHE and MODS scales and the use of medications to the risk factors after the complications from chronic gastroduodenal ulcers. So, when these adverse factors are detected in patients, erosive and ulcerative lesions of the gastrointestinal tract should be prevented.

The indicator of the mean blood pressure was of great importance. At the initial numbers up to $40 \mathrm{mmHg}$ the mortality was $35.3 \%$, at $41-60 \mathrm{~mm} \mathrm{Hg}$. $28.2 \%$, at $61-80$ $\mathrm{mmHg} 10.5 \%$, at $81-100 \mathrm{~mm} \mathrm{Hg} 3.9 \%$ and at $100 \mathrm{mmHg}$ and higher $3.8 \%$.

The total mortality rate in the group of the patients with bleeding from chronic ulcers of the stomach and duodenum was $6.1 \%$ (64 patients).

29 patients (45.3\%) of that number were hospitalized in a state of irreversible hemorrhagic shock who were in the intensive care unit from 10 minutes. up to 1 day. Despite the treatment the patients' condition got worse progressively. Apparently, we were not able to help this category of the patients that, unfortunately, allowed us to attribute them to prognostically adverse effects of the treatment. We assigned one patient who was operated on an emergency basis at the height of bleeding to the same group. Despite providing surgical treatment and infusion therapy on the operating table it was not possible to remove the patient from the state of hemorrhagic shock. Unpredictable adverse effects of the treatment included 5 patients (1.9\%). All patients underwent surgical intervention based on the indications, however, in the postoperative period pancreatic necrosis (4 patients) and pulmonary embolism (1 patient) developed.

In 31 patients $(48.4 \%)$ the direct cause of death was the decompensation of somatic pathology which developed against the background of severe posthemorrhagic anemia. All these patients did not receive adequate medication.

As can be seen from the study, the greatest impact on the development of an adverse outcome of the disease had bleeding recurrence (BR). BR was detected in 52 patients $(19.5 \%)$. As a consequence of BR the phenomenon of hemorrhagic shock developed in 23 patients (44.2\%) and it was the direct cause of their death. BR of these patients had clinical manifestations such as repeated vomiting of blood, melena, decreased blood pressure, loss of consciousness. Among them there were 8 patients $(34.8 \%)$ operated on the emergency basis, the remaining 15 patients $(65.2 \%)$ did not have any surgery. In 4 patients (7.7\%) BR led to decompensation of somatic pathology and death. In 7 patients (13.5\%) clinical manifestations were absent, however, the fact of repeated bleeding led to the need to change the treatment tactics. The forced surgical treatment that was started against the background of posthemorrhagic anemia can be considered a tactical mistake. As a consequence of it there was the development of postoperative complications in the form of insolvency of the anastomosis, DIC, etc.

Thus, BR is a major factor in the development of adverse effects of treatment. Surgical intervention performed by force against the background of severe anemia and decompensation of somatic pathology is not a method of choice and it is accompanied by high mortality. In the table above, high mortality rates during the operations at bleeding height $-18.8 \%$ and in connection with $\mathrm{BR}-15.4 \%$ are noteworthy. At the same time the mortality rate after the operations that were performed after stabilization is almost 3 times lower and in the planned order it does not reach $1 \%$. So, the main task facing the team of the specialists is the prevention of bleeding recurrence.

\section{DISCUSSION}

Several factors influence the occurrence of BR such as the degree of blood loss, topography, the size and depth of the ulcer defect, the usefulness of antisecretory drug therapy $(\mathrm{ADT})$ and endoscopic prophylaxis of bleeding recurrence (EPBR). In particular, the least likelihood of bleeding recurrence was noted with stomach ulcers less than 1.5 $\mathrm{cm}$ in diameter and with duodenal ulcers less than $1 \mathrm{~cm}$ in diameter. In this case as the size of the ulcers increases the frequency of bleeding recurrence rises. In this regard especially dangerous are giant ulcers (the stomach more than $3 \mathrm{~cm}$ in diameter, the duodenum more than $2 \mathrm{~cm}$ in diameter). The likelihood of bleeding recurrence is significantly higher with deep ulcers and especially with stenosis of the pyloroduodenal segment that is not passable for an endoscope. The latter concerns duodenal ulcers.

Currently, the use of proton pump blockers is considered a priority form of ADT. The use of these drugs according to the traditional scheme is accompanied by BR in $15.8 \%$ of the patients. The absence or inferiority of drug therapy almost doubles the risk of bleeding recurrence to $32.7 \%$. At the same time the combination of bolus and prolonged use of proton pump blockers ( $80 \mathrm{mg}$ IV bolus, then $8 \mathrm{mg} / \mathrm{h}$ for 2-3 days) reduces the risk of bleeding to $6.8 \%$.

We always began arresting of prolonged bleeding with perivasal and periulcerous administration of vasoconstrictors and procoagulants. As a rule, the introduction of the so-called "hemostatic mixture" made it possible to stop the initial continued bleeding in $75.4 \%$ of patients within 3-5 minutes from the beginning of the manipulation. $11.7 \%$ of the patients suffered from hemostasis which in result of the usage of the physiological solution created the infiltration shaft around the defect and it did not require further electro- or thermocoagulation methods. The preference was given to periulcerous injections of physiological solution or glucose solution and the application of foam or film-forming preparations. The addition of vasoconstrictors to the mixture and the use of coagulation are considered advisable only if there is a high risk of bleeding recurrence. Control endoscopic examinations were performed according to the rules adopted for chronic ulcers. 
The studies were performed every 4-12 hours until the signs of unreliable hemostasis disappeared. The higher the likelihood of bleeding recurrence the presence of a loose blood clot, large, protruding into the lumen, thrombosed vessels, hemorrhagic plaque at the bottom of the defect, the more often control studies are performed. It allows you to identify either bleeding recurrence at an earlier stage and carry out appropriate measures or perform preventive manipulations with a shorter interval between them to consolidate the result. Reducing the risk of bleeding recurrence that is determined during the control studies (the presence of small dark thrombosed vessels that do not protrude into the lumen, an increase in the layer of fibrinous plaque at the bottom of the defect) allows you to increase the interval between control studies and when performing the manipulations themselves it is possible to go to the most gentle methods - application of coagulating drugs, foaming or film-forming drugs. In this case, special attention is paid to the introduction of the drugs that create favorable conditions for more rapid relief of perifocal inflammation caused by underlying disease or resulting from previous manipulations. The absence of the signs of unreliable hemostasis is an indication for stopping endoscopic prophylaxis of bleeding recurrence.

An essential condition for the prevention of bleeding recurrence is high-quality and complete endoscopic therapy. Diagnostic errors during both the initial study and endoscopic monitoring lead to the wrong choice of treatment method. Thus, due to incorrect interpretation of BR data occurred in 17 operated patients (6.3\%). Inadequate primary endoscopic hemostasis (EH) led to early BR in 7 patients (2.6\%), inadequate EPBR at admission and during treatment monitoring led to the development of BR in 56 patients $(20.9 \%)$. In other words, the examination technology compliance, correct interpretation of the data and full-fledged manipulations would most likely help to avoid BR in $29.8 \%$ of patients. Patients with continued bleeding should be assigned to a high-risk group for developing BR. To achieve stable hemostasis, especially in the cases when profuse bleeding is noted during the initial study, it is justified to use a combination of the injection method with diathermocoagulation (DC) and thermal coagulation (TC). Such a potentiated $\mathrm{EH}$ effect is equally both effective and reliable. While performing endoscopic monitoring the preference should be given to coagulation methods of diathermo- and hydrodiatermocoagulation.

As a rule, by the time $\mathrm{EH}$ was used the bleeding was arrested, however, the achieved hemostasis was considered unreliable as a loose blood clot was formed and there was no full confidence in the absence of blood leakage. When applying coagulation techniques we tried to avoid the contact of the distal end of the probe with the deep structures and the walls of the mucosal defect in order to avoid the disturbance of the unstable state of hemostasis and the development of deep destructive changes. We began coagulation from the periphery of the clot, gradually compacting it and reducing its volume. Coagulation was combined with gentle aspiration of the loose part of the clot and liquid blood or with the washing of the contents of the ulcer crater. We consider complete aspiration of the clot to be an irrational and dangerous measure. Electrocoagulation of a clot is quite a complex and precise manipulation that requires great care when being performed because of the possibility of "welding" of the clot to the diathermosonde. Recently, we have been actively using argon-plasma coagulation which is devoid of these negative properties. The advantages of the method include the absence of deep tissue damage, reliable and rapid coagulation of the clot. It was performed in 122 patients and we were convinced of the effectiveness of this method.

\section{CONCLUSIONS}

1. The main reason for the mortality of the patients with AUGDB was decompensated hemorrhagic shock that developed at the prehospital stage in $45.3 \%$ and $44.2 \%$ of the patients as a result of BR during their treatment.

2. Inadequate drug therapy increases the risk of bleeding recurrence from 15.8 to $32.7 \%$. The use of proton pump blockers, the combination of bolus and prolonged use of proton pump blockers, allows reducing the risk of BR development (up to 6.8\%) in half. Endoscopic hemostasis with the combination of the injection method with DC or TC can be considered full-fledged and when performing endoscopic monitoring the preference should be given to coagulation methods of diathermo-and hydrodiermacocoagulation.

\section{REFERENCES}

1. Shepet'ko E.N., Efremov V.V., Ignatov A.V., Kozak S.Ju. Transformacija hirurgicheskoj taktiki i neposredstvennye rezul'taty lechenija ostryh jazvennyh duodenal'nyh krovotechenij. Russ. J. gastroenter., gepatol., koloproktol. 2013; 5:30. (in Russian).

2. Shuliarenko V.A., Mamchych V.I., Feleshtynskyi Y.P. Taktyka i perspektyvy likuvannia khvorykh z vyrazkovymy hastroduodenalnymy krovotechamy. Khirurhiia Ukrainy. 2012; 2:30-34. (in Russian).

3. Snisar A.V. Medyko-sotsialni rezultaty khirurhichnoho likuvannia vyrazkovoi khvoroby, shcho uskladnylas krovotecheiu. Med. perspektyvy. 2012;1:156-158. (in Ukrainian).

4. Botianu A., Matei D., Tantau M., Acalovshi M. Mortality and need of surgical treatment in acute upper gastroduodenal bleeding: a one year stady in a tertiary center with a 24 hours, day - 7 days, week endoscopy call. Has anything changed? Chirurgia (Bucur).2013, 312-318.

5. Marmo R., Koch M., Cipolletta L. Predicting mortality in patients with in-hospital nonvariceal upper GI bleeding: a prospective, multicenter database study. Gastrointest Endoscop. 2014; 79: 741.

6. Girelli C.M., Nardo B., Bernasconi G. A very obscure gastrointestinal bleeding. Gastroenterology. 2012; 142(4): 3-4.

7. Tsoi K.K., Chiu P.W., Chan F.K. The risk of peptic ulcer bleeding mortality in relation to hospital admission on holidays: a cohort study on 8,222 cases of peptic ulcer bleeding. Am. J. Gastroenterol. 2012; 107: 405.

8. Laine L., Jensen D.M. Management of patients with ulcer bleeding. Am. J. Gastroenterol. 2012; 107:345.

9. Abraham N.S. Novel oral anticoagulants and gastrointestinal bleeding: a case for cardiogastroenterology. Clin. Gastroenterol. Hepatology. 2013;11(4):324-328. 
ORCID and contributionship:

Gennady E. Samoilenko: 0000-0003-0075-4307 A, E, F

Stanislav O. Zharikov: 0000-0001-6503-1068 ${ }^{\mathrm{C}}$

Ruslan P. Klimanskyi: 0000-0002-9118-7813 ${ }^{B, D}$

\section{Conflict of interest:}

The Authors declare no conflict of interest.

\section{CORRESPONDING AUTHOR}

Ruslan P. Klimanskyi

Donetsk National Medical University

39 Machinostroiteley st., 84313 Kramatorsk, Ukraine

tel: +380509038996

e-mail: ruslanmozg@gmail.com

Received: 08.11.2019

Accepted: 30.06 .2020

A - Work concept and design, B - Data collection and analysis, C - Responsibility for statistical analysis,

$\mathbf{D}$-Writing the article, $\mathbf{E}$-Critical review, $\mathbf{F}$ - Final approval of the article 